\title{
Myopathy as the Initial Manifestation of Primary Amyloidosis
}

\author{
J. Keith, Z. Afshar-Ghotli, R. Roussev, B. Ernst, B. Young, J.M. Bilbao
}

Can. J. Neurol. Sci. 2011; 38: 161-164

\section{CASe 1}

A 63 year-old man had a past medical history of diabetes and hypertension, did not smoke or drink alcohol, and had no relevant family history. Over the course of several months he developed left leg weakness and difficulty climbing stairs. He denied other symptoms including fever, sweats, chills, shortness of breath or chest pain. On general examination he had no adenopathy or hepatosplenomegaly. Neurological examination revealed focal weakness of the left hip flexor but was otherwise normal. Electromyography (EMG) showed a myopathic process. A biopsy was performed on an unspecified leg muscle.
On routine staining with Hematoxylin Phloxine Saffron (HPS) the skeletal muscle biopsy showed amyloid throughout the perimysium and more focally within the endomysium, characterized by amorphous material juxtaposed against the connective tissue (Figure 1A). The amyloid stained strongly with Puchler's Alkaline Congo Red (Figure 1B) and appeared green by polarized microscopy (Figure 1C). There were also myopathic abnormalities including scattered necrotic fibres and internalization of myonuclei (Figure 1D). Groups of atrophic fibres were noted, as was a paucity of microvasculature, and no perifascicular atrophy was noted. Plastic embedded sections and

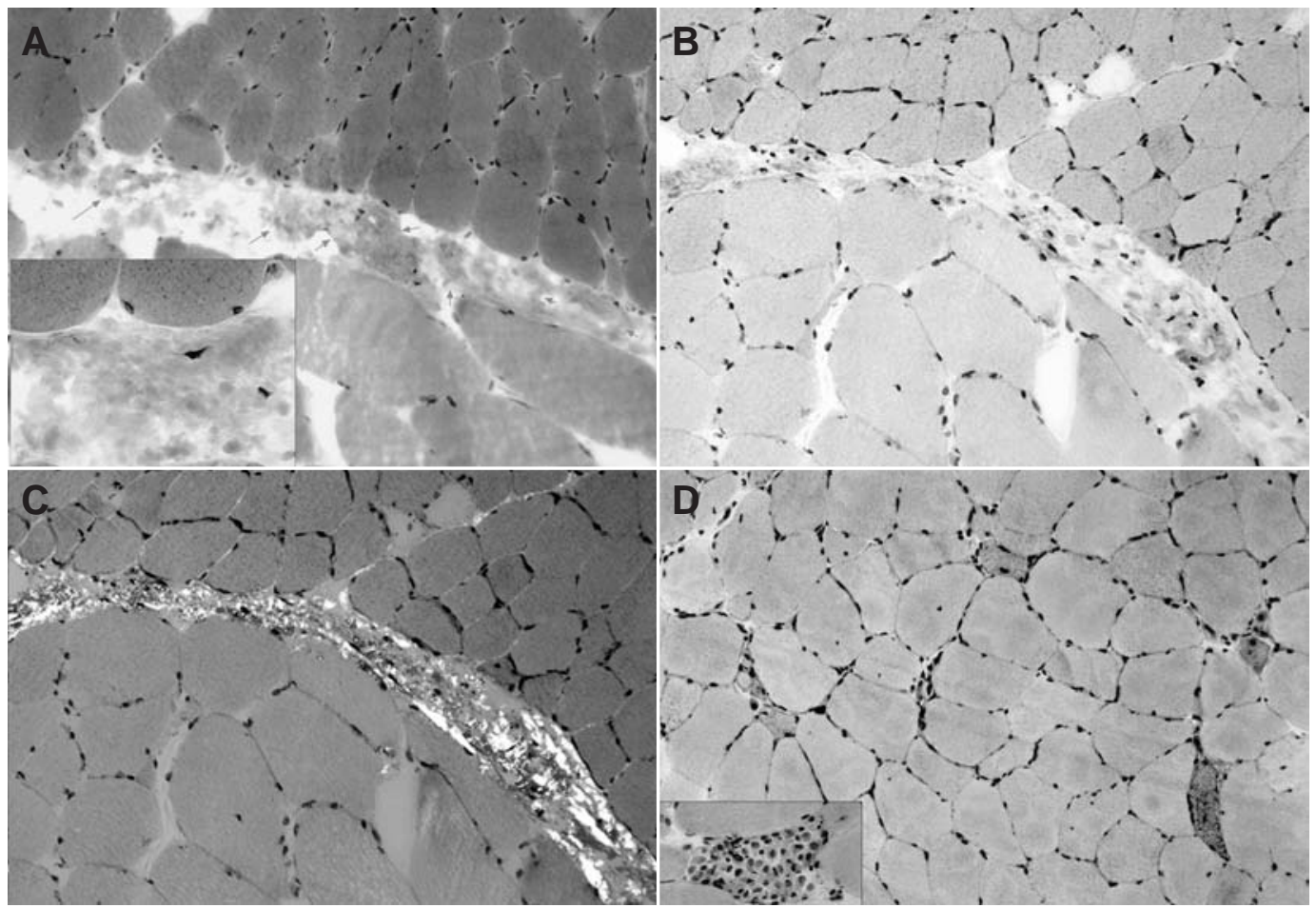

Figure 1: (A) Amorphous material, amyloid, juxtaposed with connective tissue in the perimysium (Hematoxylin Phloxine Saffron); Amyloid within the perimysium (B) highlighted by Congo Red staining and appearing green upon polarized microscopy (C). (D) Myopathic abnormalities (Hematoxylin Phloxine Saffron).

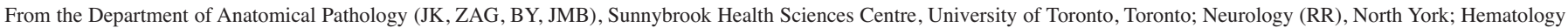
(BE), Credit Valley Hospital, Mississauga, Ontario, Canada.

Received May 13, 2010. Final Revisions Submitted July 20, 2010

Correspondence to: Julia Keith, Department of Anatomical Pathology, Sunnybrook Health Sciences Centre, Room E4-32, 2075 Bayview Avenue, Toronto, Ontario,

M4N 3M5, Canada. 


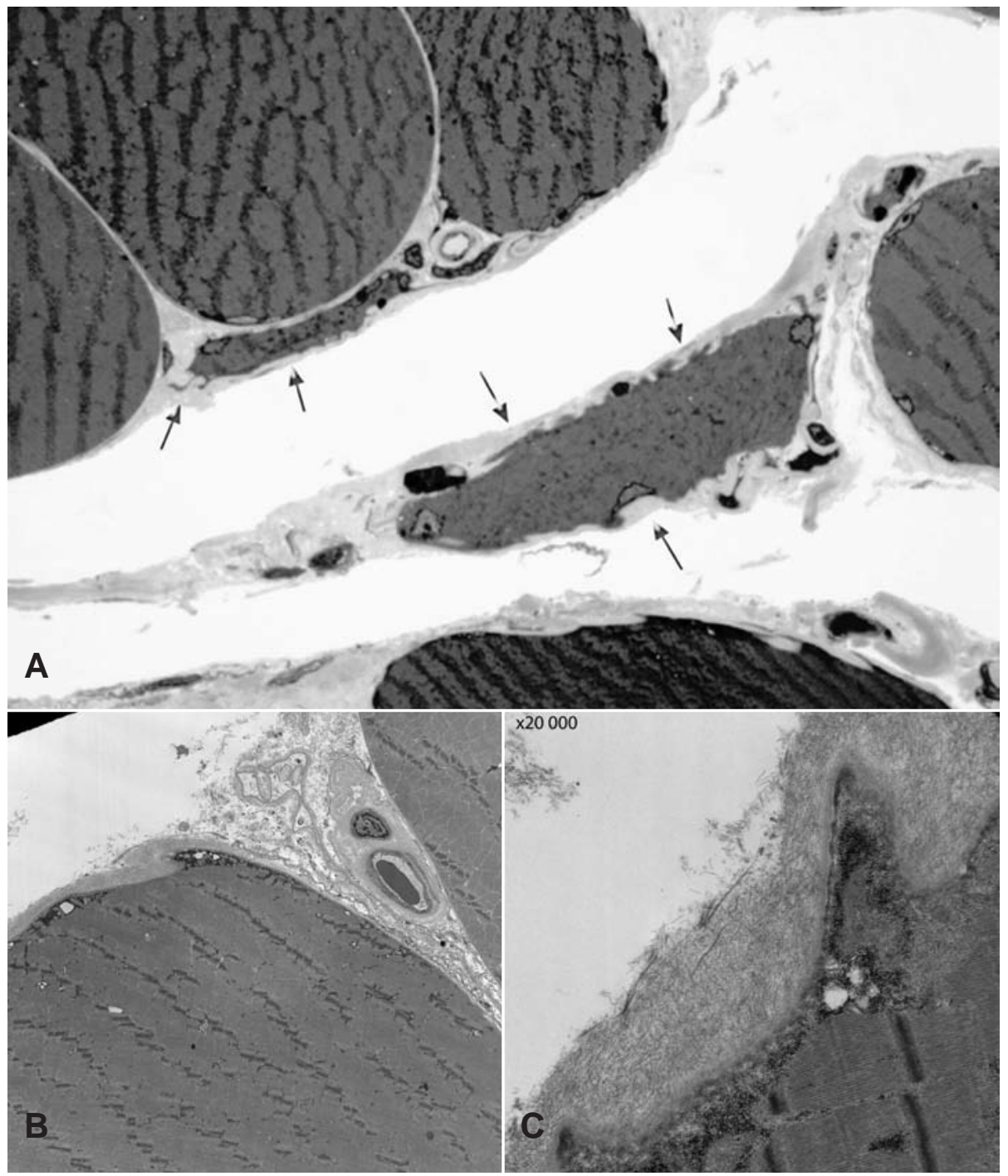

Figure 2: Amyloid fibrils adjacent to the myocyte sarcolemma and surrounding the vasculature on ultrastructural examination.

electron microscopy showed extracellular amyloid opposed to the sarcolemma (Figure 2A, 2B), disrupting but not breaching the basal lamina, and surrounding the vasculature (Figure 2B, $2 \mathrm{C})$ and the mitochondria appeared normal. Using immunofluorescence strong lambda light chain staining (DAKO monoclonal antibody, 1:400 dilution) was noted in the interstitium, colocalizing with the collections seen on Congo Red.

Additional investigations included normal serum protein electrophoresis, an elevated creatine kinase (CK) of $694 \mathrm{U} / \mathrm{L}$ (normal 5-30 U/L), and many normal studies such as complete blood count, anti-DNA, anti-cyclic citrullinated protein antibodies (anti-CCP), anticardiolipin, microalbumin, serum $\mathrm{B} 12$, prostate-specific antigen (PSA), and thyroid-stimulating hormone (TSH). Computed tomogram scan of the abdomen and pelvis showed enlarged lymph nodes. Echocardiogram showed concentric cardiac hypertrophy, likely secondary to hypertension. A 24-hour urine collection contained 1.6 grams of lambda light chain (Bence Jones) monoclonal protein. A bone marrow biopsy revealed amyloid deposition within the vessels and stroma, but no evidence of a plasma cell dyscrasia.

He was treated with Melphalan and Prednisone and was not considered a candidate for bone marrow transplantation. The patient left the country and further follow-up is not available.

\section{CASE 2}

A 69 year-old male had a past medical history of diabetes, hypertension, coronary artery disease requiring coronary angioplasty and stenting, congestive heart failure, as well as restrictive respiratory disease. Other relevant history included elevated liver enzymes with a normal liver on MRI, and a spinal MRI showing synovial cysts at L5/S1, accounting for a history 
of lower extremity polyradiculopathy. At the time of presentation he had been on a statin for five years. His family history was unremarkable. His presenting neuromuscular complaint was proximal muscle weakness for the past three years, impairing his ability to raise his arms and climb stairs. He denied sensory phenomena or other neurological symptoms. Neurological examination showed mild weakness of the deltoid muscle, reduced vibration sense asymmetrically and diminished symmetrical reflexes. Electrodiagnostic studies revealed mild myopathic phenomena (trace fibrillations and increased polyphasic units in the deltoid), normal motor conduction velocities, and slightly reduced conduction velocity of the sural nerve. Investigations revealed an elevated CK of $300 \mathrm{U} / \mathrm{L}$ (normal 5-30 U/L). His statin therapy was discontinued. Several months later his weakness had persisted and his CK had risen to 490 U/L. Repeat electrodiagnostic studies confirmed a myopathic process as there was decreased motor unit action potential amplitudes and duration plus polyphasic units. A muscle biopsy was requested.

A left deltoid skeletal muscle biopsy showed a combination of myopathic features, including internalization of myonuclei and myonecrosis, and neurogenic atrophy (Figure 3A) characterized by groups of atrophic fibres and like-fibre type grouping using immunohistochemical staining for fast and slow twitch myosin. No perifascicular atrophy was noted and there were no mitochondrial abnormalities. Amyloid deposition within the endomysium, extensively within the vasculature (Figure 3B, $3 \mathrm{C}$ ), and within a single intramuscular nerve twig was identified by Puchler's Alkaline Congo Red stain which was green on birefringence. Using immunofluorescence there was strong immunolabeling for lambda light chains (DAKO monoclonal antibody, 1:400 dilution).

Subsequent serum protein electrophoresis showed a gamma globulin paraprotein, further characterized as IgG type lambda on serum immunofixation electrophoresis. A bone marrow biopsy was hypercellular and contained 30\% plasma cells, consistent with plasma cell dyscrasia.

\section{Discussion}

Amyloid is not a chemically distinct substance, but is rather a term encompassing any insoluble fibrillar protein which assumes beta-pleated sheet architecture and accumulates extracellularly in either a localized or diffuse fashion, causing disruption of tissue structure and function, in a variety of clinical settings. The intracellular sarcoplasmic deposition of amyloid seen in Inclusion Body Myositis is an exception to this scenario. At least 25 amyloidogenic proteins have been identified $^{1-3}$, the most common of which are immunoglobulin light chains (termed AL or 'amyloid light chain'), AA amyloid ('amyloid associated' which occurs in the context of infectious or inflammatory diseases), and familial forms. Primary amyloidosis refers to a diffuse distribution of predominantly lambda light chain amyloid, often in the context of a plasma cell dyscrasia, and has an incidence of more than ten per million person-years in the United States ${ }^{3}$. Primary amyloidosis has a median survival of one to two years, with kidneys being the most frequently affected $\operatorname{organ}^{1}$, and more than half of patients die of cardiac disease ${ }^{3}$. Management strategies including Melphalan, steroids, other immunomodulating agents and bone marrow transplant all aim

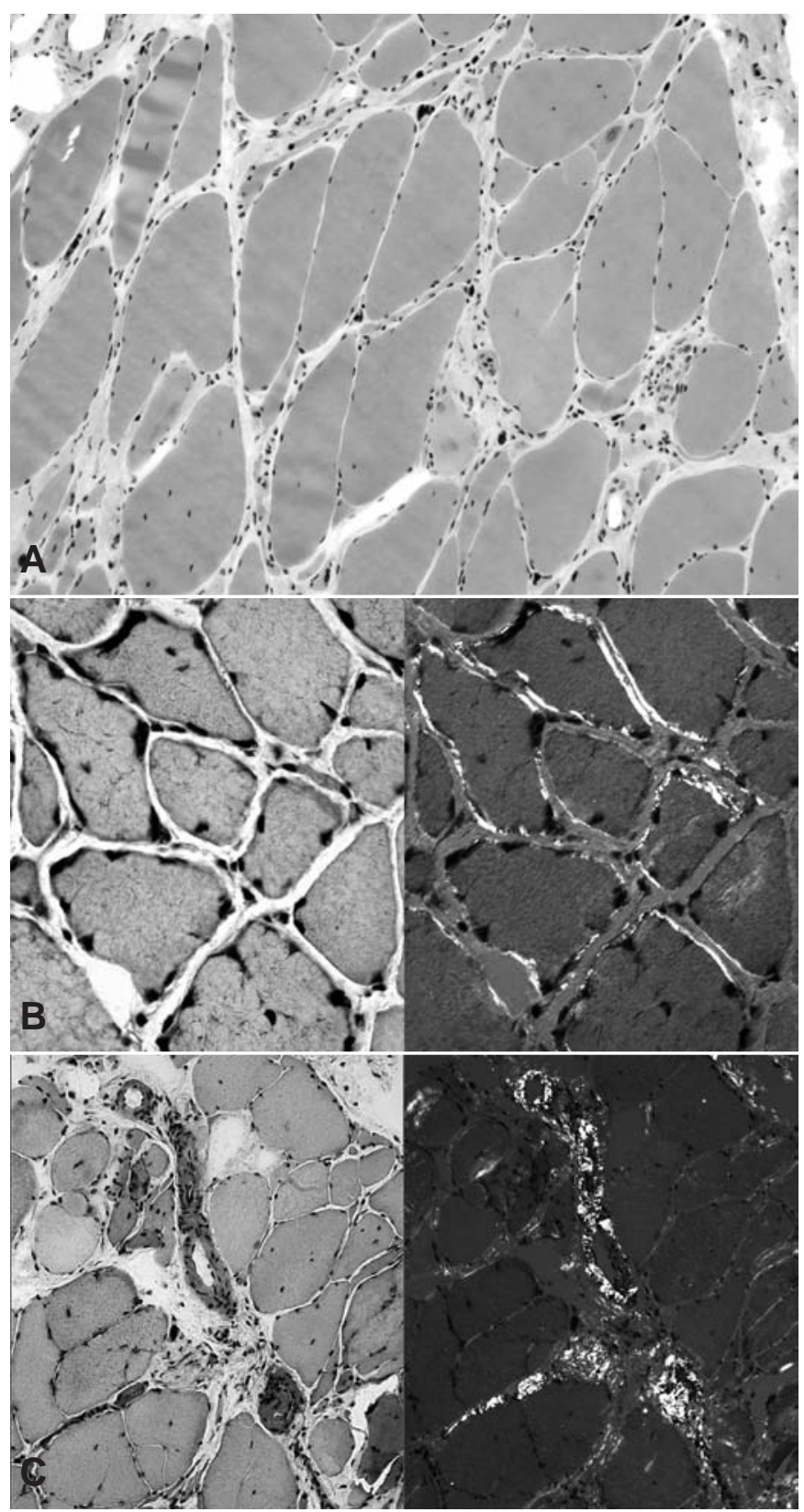

Figure 3: (A) Myopathic and neurogenic abnormalities on deltoid muscle biopsy (Hematoxylin and Eosin); (B,C) Amyloid deposition in the endomysium and vasculature (Congo Red) appearing green upon polarized microscopy.

to eliminate the supply of the amyloidogenic protein ${ }^{1}$, and early treatment of amyloidosis is associated with improved outcome and survival as amyloidogenesis and deposition can be halted and, in some cases, reversed ${ }^{1}$. As the diagnosis of amyloidosis relies on the demonstration of amyloid deposits in tissue ${ }^{1}$, the importance of its detection in biopsy samples is paramount.

Seventeen percent of patients with primary amyloidosis have peripheral neuropathy ${ }^{4}$, and amyloid is most frequently encountered in neuropathology practice in peripheral nerve 
biopsies as endoneurial, perivascular deposits in the context of either familial amyloid neuropathy (usually due to transthyretin mutations), or in gammopathy, with resulting loss of small myelinated and unmyelinated axons ${ }^{4}$.

Our two cases serve as reminders of amyloid's ability to initiate a myopathic process. Seventy-nine previously reported cases of amyloid myopathy were reviewed by Chapin and the most common presenting symptom was proximal muscle weakness ${ }^{5}$. Rare examples of severe respiratory failure due to amyloid deposition in the diaphragm muscle are noteworthy ${ }^{6}$. As shown by our cases, other signs and symptoms of systemic amyloidoses may or may not be present when muscle weakness appears. Congestive heart failure has been documented in $15 \%$ of amyloid myopathy cases ${ }^{5}$, and amyloidosis may have been a contributing factor to the congestive heart failure of Case 2. The classic amyloidosis clinical stigmata of macroglossia and/or muscle pseudohypertrophy were not present in our cases, and were noted in only $34 \%$ of the cases Chapin reviewed ${ }^{5}$. Most patients with amyloid myopathy have primary AL amyloidosis $^{5,7}$, and half have a plasma cell dyscrasia or multiple myeloma. The prognosis of amyloid myopathy is generally poor with a mean survival of 22 months, but response to immunomodulating therapies is variable and patients with long survival, resolution of congestive hart failure, and eradication of light chains from urine are on record ${ }^{5,8-10}$.

The histopathology of our two cases is similar to those reviewed by Chapin, where vascular and/or interstitial deposition of amyloid was universal (with deposition limited to the vasculature in 15\%), denervation atrophy was noted in $38 \%$ of cases and $23 \%$ had necrotic/regenerating fibres ${ }^{5}$. Importantly, our cases illustrate that the presence of amyloid on routinely stained sections, especially H\&E, can be subtle, and in fact $24 \%$ of amyloid myopathy cases are initially misdiagnosed on biopsy ${ }^{5}$. This notion supports our suspicion that Congo Red staining should be included in a routine histology panel for muscle biopsies, and polarization of the slide is encouraged. It is especially prudent to search for the presence of amyloid in any muscle biopsy with denervation atrophy and/or myonecrosis for which no alternative explanation such as an inflammatory myopathy is seen. Despite the emergence of newer immunohistochemical techniques for subtyping amyloid deposits, which must be interpreted with caution, Congo Red staining remains the gold standard for the detection of amyloid in tissue ${ }^{1}$.

The mechanism of myocyte injury and myonecrosis in the setting of amyloidosis remains elusive. It was originally suggested that myocytes may be surrounded and mechanically compressed by extracellular amyloid, but subsequent studies did not support this notion ${ }^{11}$. Ultrastructural studies have suggested that deposited amyloid does not pierce the sarcolemma ${ }^{12}$ and the ultrastructural findings in our case support this notion. Both ultrastructural and TUNEL studies have shown that muscle injury is not due to amyloid induced apoptosis ${ }^{11}$. The role of the complement cascade, assessed via immunolabeling with C5b9 membrane attack complex, is uncertain, with some studies reporting no immunolabeling ${ }^{11}$ and others showing complement deposition colocalizing with amyloid ${ }^{13}$. We believe that myocyte injury in amyloidosis may be multifactorial, including ischemia ${ }^{11}$ (supported by the focal reduction in the number of capillaries seen in our cases), interference with the diffusion of nutrients through the sarcolemma ${ }^{11}$, and/or denervation. It is possible that our patients' diabetes may have been a contributing factor in the development of neurogenic atrophy.

In conclusion, myopathy is an uncommon initial manifestation of primary amyloidosis, but pathologists should entertain this entity and include Congo Red in the muscle biopsy protocol in cases with unexplained myopathic abnormalities, despite the absence of other systemic signs of amyloidosis.

\section{REFERENCES}

1. Picken MM. Amyloidosis - where are we now and where are we heading? Arch Pathol Lab Med. 2010; 134:545-51.

2. Westermark P, Benson MD, Buxbaum JM, et al. Amyloid protein fibril nomenclature-2002. Amyloid. 2002; 9:197-200.

3. Desai HV, Aronow WS, Peterson SJ. Cardiac amyloidosis: approaches to diagnosis and management. Cardiol Rev. 2010; 18:1-11.

4. Bilbao JM, Schmidt R, Hawkins C. Diseases of peripheral nerve. In: Love S, Louis DN, Ellison DW, editors. Greenfield's Neuropathology, 8th ed. London: Hodder-Arnold; 2008. p.1675-81.

5. Chapin JE, Kornfeld M, Harris A. Amyloid myopathy: characteristic features of a still underdiagnosed disease. Muscle Nerve. 2005; 31:266-72.

6. Ashe J, Borel CO, Hart G, Humphrey RL, Derrick DA, Kunel RW. Amyloid myopathy presenting with respiratory failure. J Neurol Neurosurg Psychiatry. 1992; 55:162-5.

7. Nadkarni N, Freimer M, Mendell JR. Amyloidosis causing a progressive myopathy. Muscle Nerve. 1995; 18:1016-8.

8. Gertz MA, Kyle RA. Myopathy in primary systemic amyloidosis. J Neurol Neurosurg Psychiatry. 1996; 60:655-60.

9. Metzler JP, Fleckenstein JL, White CL, Haller RG, Frenkel EP, Greenlee RG. MRI evaluation of amyloid myopathy. Skeletal Radiol. 1992; 21:463-5.

10. Sheehan-Dare RA, Simmons AV. Amyloid myopathy and myeloma: response to treatment. Postgrad Med J. 1987; 63 (736):141-2.

11. Spuler S, Emslie-Smith A, Engel AG. Amyloid myopathy: an underdiagnosed entity. Ann Neurol. 1998; 43:719-28.

12. Jennekens FG, Wokke JH. Proximal weakness of the extremities as a main feature of amyloid myopathy. J Neurol Neurosurg Psychiatry. 1987; 50:1353-8.

13. Rodolico C, Mazzeo A, Toscano A. Amyloid myopathy presenting with rhabdomyolysis: evidence of complement activation. Neuromuscul Disord. 1996; 16:514-7. 